\title{
Fatores determinantes do controle da criminalidade em gestão de políticas de segurança pública
}

\author{
Sabrina Oliveira de Figueiredo ${ }^{1}$ \\ Larissa Alves Sincorá 1 \\ Maria Clara de Oliveira Leite 1 \\ Marcelo Moll Brandão 1
}

1 Universidade Federal do Espírito Santo / Programa de Pós-Graduação em Administração, Vitória / ES - Brasil

\begin{abstract}
É alvo central de discussões a temática relacionada à segurança pública no país, especialmente, no tocante aos índices de criminalidade. Este estudo aborda a temática a partir da Teoria Econômica da Escolha Racional e de teorias de base sociológica que buscam explicar as causas do crime. O objetivo do estudo foi identificar fatores determinantes para o controle dos índices de criminalidade a partir de dados do estado do Espírito Santo. Trata-se de um estudo de abordagem quantitativa. Foram utilizados dados secundários, tendo como referência o modelo teórico proposto. Adotou-se, ainda, como método de teste de hipóteses, o modelo de regressão linear, com o auxílio do software SPSS Statistics, versão 25. Os resultados da pesquisa demonstram que o elemento relativo à empregabilidade da população influencia direta e significativamente o controle dos índices de crimes de homicídios dolosos e de crimes contra o patrimônio. Ademais, conjugado ao emprego (atividades laborais formais), os fatores renda e educação da população demonstraram influenciar positivamente no controle dos crimes contra o patrimônio.

Palavras-chave: fatores determinantes; controle da criminalidade; homicídios dolosos; crimes contra o patrimônio; segurança pública.
\end{abstract}

\section{Determinantes del control de la criminalidad en la gestión de políticas de seguridad pública}

La temática relacionada con la seguridad pública en el país es objeto central de discusión, especialmente con respecto a los índices de criminalidad. Este estudio aborda el tema a partir de la teoría económica de la elección racional y las teorías sociológicas que buscan explicar las causas del delito. El objetivo del estudio fue identificar los factores determinantes para el control de los índices de criminalidad con base en datos del estado de Espírito Santo. Este es un estudio con un enfoque cuantitativo. Se utilizaron datos secundarios, basados en el modelo teórico propuesto. El modelo de regresión lineal también se adoptó como método de prueba de hipótesis, con la ayuda del software SPSS Statistics versión 25. Los resultados de la investigación muestran que el elemento relacionado con la empleabilidad de la población influye directa y significativamente en el control de los índices de homicidios dolosos y delitos contra la propiedad. Además, combinado con el empleo (actividades laborales formales), los factores ingresos y educación de la población han demostrado tener una influencia positiva en el control de los delitos contra la propiedad.

Palabras clave: factores determinantes; control de la criminalidad; homicidios dolosos; delitos contra la propiedad; seguridad pública.

\section{Determinants of crime control in public security policy management}

The topic related to public security in Brazil is central in discussions regarding crime rates. This study approaches the theme from the economic theory of rational choice and sociological theories that seek to explain the causes of crime. The study's objective was to identify determining factors for controlling crime rates based on data from the state of Espírito Santo. This is a study with a quantitative approach. Secondary data were used, based on the proposed theoretical model. The linear regression model was also adopted as a hypothesis testing method, with the aid of the SPSS Statistics software version 25. The results show that the element related to the population's employability directly and significantly influences the control of crime rates, intentional homicides, and crimes against property. Furthermore, combined with employment (formal labor activities), the population's income and education positively influence the control of crimes against property.

Keywords: determining factors; control of crime; willful murders; crimes against property; public security. 


\section{AGRADECIMENTOS}

Agradecemos à Coordenação de Aperfeiçoamento de Pessoal de Nível Superior (CAPES) pelo suporte financeiro para execução desta pesquisa.

\section{INTRODUÇÃO}

A temática relacionada à violência e à criminalidade no país, incluindo os fatos ocorridos de repercussão nacional que envolvem assassinatos, roubos seguidos de morte, assaltos e outros crimes são constantemente alvos de discussão política, acadêmica, midiática e da sociedade em geral. De acordo com estudo técnico realizado e divulgado no ano de 2018 pelo Fórum Brasileiro de Segurança Pública (FBSP), o Brasil registrou, em 2017, 63.880 mortes violentas intencionais (mortes decorrentes de homicídios dolosos, latrocínios, lesões corporais seguidas de morte e de confrontos policiais), o que equivale a 175 mortos por dia.

A taxa de homicídios dolosos para cada grupo de 100 mil habitantes - índice usualmente utilizado para comparar os níveis de criminalidade entre unidades geográficas -, no ano de 2017, no país, foi de $30,8 \%$, que significou um acréscimo de cerca de 3\% se comparado com o índice registrado em 2016 (FBSP, 2018). Também deve-se registrar que o Brasil concentra cerca de 14\% dos homicídios ocorridos no mundo e, por conta dessa estatística, o país inclui-se entre os $10 \%$ do conjunto de países que possuem as maiores taxas de homicídio (Secretaria-Geral da Presidência da República [SGPR], 2018). A taxa de homicídios do Brasil é semelhante às taxas apresentadas em países africanos, como Ruanda, África do Sul e República Democrática do Congo (SGPR, 2018).

Quando se trata dos custos econômicos da violência do Brasil, os índices são ainda mais alarmantes. Em 2017, o financiamento da política nacional de segurança atingiu o patamar de 84,7 bilhões, o que denota que o país gastou $\mathrm{R} \$ 408,13$ por cidadão brasileiro com segurança pública (FBSP, 2018). Observou-se que, entre 1996 e 2015, ocorreu um incremento de gastos em segurança pública, saindo de um patamar de 32 bilhões para 90 bilhões de reais gastos por ano, respectivamente (SGPR, 2018). Interessante, ainda, ressaltar que os gastos em segurança pública não são proporcionalmente realizados entre as esferas federativas (Afonso, 2017; SGPR, 2018; FBSP, 2018). Do total despendido em segurança entre 1996 e 2015, cerca de 19\% equivaleram a gastos da União, enquanto a participação dos municípios chegou a apenas 6\% dos gastos (SGPR, 2018). Em 2017, a União gastou 9,7 bilhões em segurança pública, enquanto os estados e os municípios despenderam 69,8 bilhões e 5,1 bilhões, respectivamente (FBSP, 2018).

Entre as mais diversas discussões relacionadas à criminalidade, surgem aspectos complexos e desafios a serem perseguidos pela política nacional (em todas as suas esferas político-administrativas) e pelos órgãos públicos responsáveis pela segurança. De fato, a atribuição dos órgãos responsáveis pela segurança pública não é uma missão fácil: a preservação da ordem pública e da incolumidade das pessoas e do patrimônio (Constituição [da] República Federativa do Brasil, 1988).

A eficácia da política pública de segurança pode não estar atrelada somente ao aumento indiscriminado do volume de gastos com o setor, mas à aplicação de recursos em ações efetivas e de impacto a médio e longo prazos. Nesta perspectiva, ganha destaque a realização de pesquisas de cunho científico que apontem quais fatores (estruturais, econômicos e sociais) são contundentes para o controle da criminalidade. Adicionalmente, a comunidade científica que investiga a área de segurança pública do país defende a expansão de estudos baseados em evidências empíricas (Afonso, 2017).

REVISTA De Administração PÚblica ｜ Rio de Janeiro 55(2): 438-458, mar. - abr. 2021 
Em linhas gerais, podem ser citadas duas principais abordagens no que tange à temática da criminalidade para fins desta pesquisa. A primeira delas, a Teoria Econômica da Escolha Racional (Becker, 1968; Ehrlich, 1973), dedica-se a explicar o comportamento criminoso como uma escolha consciente do indivíduo, o qual opta (ou não) pelo crime após realizar um balanceamento dos trade-offs envolvidos - os custos da prática criminosa e seus benefícios. Já a segunda abordagem, de cunho sociológico, engloba um conjunto de teorias, são elas: a Teoria da Associação Diferencial (Sutherland, 1973), a Teoria do Controle Social (Agnew, 1991) e a Teoria da Desorganização Social (Shaw \& McKay, 1942). Cada teoria sociológica possui particularidades, no entanto, em congruência, argumentam que o comportamento criminoso deriva do contexto e das situações da vida social.

Diante de uma série de trabalhos desenvolvidos e de distintos resultados alcançados - detalhados na fundamentação teórica adiante -, a proposta deste estudo é motivada pela expectativa de contribuir na identificação e na análise de fatores determinantes para o controle dos índices de criminalidade, a partir da realização de um estudo com emprego do método hipotético-dedutivo e da abordagem quantitativa. Portanto, partindo-se das duas principais abordagens criminológicas elencadas nesta introdução, o artigo busca responder ao seguinte problema de pesquisa: Quais os fatores determinantes para o controle dos indices de criminalidade no estado do Espírito Santo?

A contribuição teórica do estudo consiste na busca por promover o avanço nas pesquisas acadêmicas com ênfase na identificação de elementos-chave (fatores) relacionados com as circunstâncias do crime, tendo como bases teóricas dois diferentes arcabouços aplicados à Administração Pública e, mais propriamente, à área da segurança pública: a Teoria Econômica da Escolha Racional e o conjunto de teorias sociológicas formado pela Teoria da Associação Diferencial, Teoria do Controle Social e Teoria da Desorganização Social. Em seu aspecto prático, pode-se mencionar que o desenvolvimento de estudos, nessa perspectiva, tem o potencial de se adicionarem às discussões sociais em evidência e, notadamente, de subsidiar políticas na área da segurança pública e de gestão organizacional do setor, mediante evidências empíricas que identifiquem os fatores que envolvem as circunstâncias do crime.

Cabe ainda registrar que o interesse da pesquisa pelo Espírito Santo concede maior relevância à proposta, em razão da segurança pública no referido estado representar uma área de governo crítica e sensível. Durante seis anos consecutivos (2007-2012), o estado se manteve no segundo lugar do ranking nacional da taxa de homicídios dolosos, tendo alcançado em 2009 a taxa de 56,9 homicídios por 100 mil habitantes (a maior taxa de homicídios da série histórica, superior ao dobro da taxa média do país naquele ano) (Instituto de Pesquisa Econômica Aplicada [IPEA], 2018). Segundo o IPEA, a partir do ano de 2010 o estado passou a apresentar reduções consecutivas das taxas de homicídios dolosos e atingiu, no ano de 2016, o sétimo ano consecutivo de redução dos índices de homicídios. Ainda segundo o IPEA, se comparadas as taxas de homicídios dolosos no estado no ano de 2009 e 2016, observou-se uma redução considerável, na ordem de 44\%.

Isto posto, na sequência, é apresentada a fundamentação teórica que deu suporte ao desenvolvimento das hipóteses. Na terceira parte, são apresentados os aspectos do percurso metodológico e, na quarta, a apresentação dos dados e a discussão dos resultados. Por fim, tem-se a seção das conclusões, onde estão descritas as implicações dos achados do estudo, suas limitações e proposta de questões que nortearão futuras possibilidades de investigação. 


\section{FUNDAMENTAÇÃO TEÓRICA}

Em um cenário complexo como o da segurança pública, muitas são as soluções proferidas que objetivam auxiliar no combate à violência e interferir sobre as consequências dos atos criminosos, dentre as quais podem-se citar as pautas que envolvem a redução da maioridade penal (Silva \& Oliveira, 2015), a liberação do porte de armas de fogo para a população (Alessi, 2018), a extinção da progressão do regime de pena (Barbosa, 2017), além dos discursos sobre a ampliação de investimentos em torno do reequipamento das polícias estaduais a partir da contratação de mais servidores, aquisição de armamentos, viaturas e outros materiais. Na contramão das soluções pautadas no imediatismo e de outras que atuam no contexto pós-crime, as bases teóricas têm um papel essencial, tendo em vista a identificação dos fatores criminogênicos que explicam as causas dos crimes, quer sejam fatores do plano individual, como disfunções biológicas e psíquicas, fatores provenientes das relações sociais e fatores estruturais de ordem social, econômica e demográfica (Cerqueira, 2014; Cerqueira \& Lobão, 2004; Cerqueira \& Moura, 2019; Lima, Santos, Dal'Col \& Silva, 2017).

Neste presente estudo, as bases teóricas terão papel essencial em razão do histórico de pesquisas desenvolvidas, da relevância contributiva e do volume de estudos realizados. Priorizaram-se duas abordagens, as quais serão brevemente explicitadas. A primeira delas, de base clássica, refere-se à Teoria Econômica da Escolha Racional (Becker, 1968; Ehrlich, 1973) que se concentra em explicar o comportamento criminoso como uma escolha consciente do indivíduo, o qual faz uma avaliação do benefício (recompensas) e dos custos (riscos) em praticar a atividade criminosa. A segunda delas, de base sociológica, inclui uma série de teorias - são elas, Teoria da Associação Diferencial, Teoria do Controle Social e Teoria da Desorganização Social - que explicam o fenômeno do crime considerando fatores estruturais e sociais e relações interpessoais.

Pela lente da Teoria Econômica da Escolha Racional, a prática de um crime assume uma capacidade racional e deliberativa dos sujeitos, que escolhem cometer um crime pautados pelo princípio da utilidade (Shon \& Barton-Bellessa, 2015). A causa do crime estaria, assim, relacionada, de um lado, com fatores relativos à renda, como o salário percebido no mercado de trabalho formal, o desemprego e a desigualdade de renda, e de outro lado, fatores dissuasórios, como a (in)eficácia do trabalho das forças policiais e a punibilidade ou impunidade diante dos atos criminais (Cerqueira, 2014).

Muito embora essa teoria tenha trazido importantes contribuições para as Ciências Sociais, tenha se tornado proeminente em áreas diversas, como Economia, Sociologia, Ciência Política, Psicologia, e continue a despertar interesse dentro da Criminologia, os teóricos nutrem um particular ceticismo quanto a ela no que diz respeito ao pressuposto generalista e à capacidade que ela teria, por si só, de oferecer uma base teórica geral para explicar a conduta criminosa. É uma teoria cujas lacunas são citadas por vários estudiosos, segundo os quais, apesar da importância das diferenças individuais na escolha racional, a base de conhecimento sobre o assunto é limitada (Loughran, Paternoster, Chalfin \& Wilson, 2016; Ray, Baker \& Caudy, 2020).

Para Loughran et al. (2016, p. 86), por exemplo, o ceticismo em relação à teoria foi alimentado por sua generalidade. Isso porque "uma teoria que enfatiza a ponderação racional dos custos e benefícios das ações pode ser perfeitamente aplicável às decisões do mercado financeiro, mas não ao comportamento criminoso". Ademais, caso aplicável ao crime, limitar-se-ia a explicar atos criminosos contra a propriedade. Assim, somente essa teoria seria insuficiente para explicar, na prática, crimes violentos ou decorrentes de ações carregadas de forte afeto. Outra crítica é que a teoria apresenta 
um escopo conceitual limitado, ao restringir o debate aos custos financeiros e aos ganhos obtidos com a infração. Nesse sentido, Cerqueira e Moura (2019) expressam dúvidas quanto à associação dos argumentos da teoria sobre os crimes violentos, como os homicídios, uma vez que a letalidade pode ser um efeito de uma ação premeditada desvinculada de qualquer motivação econômica. Os criminologistas sociológicos seriam frequentemente hostis à teoria por sua lógica financeira (Loughran et al., 2016).

Ray et al. (2020), por sua vez, propuseram-se a testar se a psicopatia modera a relação entre riscos e recompensas de agressões autodeclaradas. Para tanto, utilizaram dados em painel, coletados a partir de uma amostra de infratores juvenis graves e de modelos híbridos estimados. Conforme apontam, até o momento (2020), não teria sido realizada pesquisa para examinar o efeito moderador da psicopatia sobre a percepção de riscos e recompensas em condutas criminais autodeclaradas. Os resultados do estudo podem ajudar a preencher uma lacuna na Teoria da Escolha Racional sobre a extensão em que as percepções de riscos e recompensas são condicionadas por diferenças individuais.

No entendimento do presente artigo, em consonância com a visão da Criminologia sociológica, para aplicar aspectos da escolha racional à literatura empírica, torna-se importante harmonizar essa perspectiva com teorias sociais do crime.

Ainda sobre a Teoria da Escolha Racional, argumenta-se que sua base clássica e contemporânea tenha fundamentado os trabalhos de Alfred Adler, um dos pioneiros na Psicologia a desenvolver uma teoria capaz de explicar as condutas criminosas (Shon \& Barton-Bellessa, 2015). Adler segue a vertente da escolha racional e afirma adicionalmente que variáveis como tempo e espaço, que influenciam as oportunidades de participar de atos ilícitos, são importantes no entendimento do fenômeno, para além de diferenças individuais de sujeitos com propensão à criminalidade com base em aspectos do self. Apesar de as teorias psicológicas não terem sido tão predominantes quanto as de cunho sociológico e as da perspectiva clássica, aventa-se o seu potencial em permitir teorizações frutíferas à Criminologia (Shon \& Barton-Bellessa, 2015).

Shon e Barton-Bellessa (2015) esclarecem que o caminho teórico de Adler aponta para uma conduta criminosa premeditada, relacionada a ganhos materiais e vinculada à falta de laços sociais, de sentimentos de pertencimento e à sensação de um tratamento injusto por aqueles ao redor. Criticase essa perspectiva por adotar uma definição limitada de crime, por - inconscientemente - apontar a lente criminológica para classes desfavorecidas, por se revelar instrumentalista e por falhar na integração com a economia política do crime em seus esforços teóricos, ao negligenciar o impacto da espontaneidade no processo e de aspectos socioeconômicos no desenvolvimento da personalidade e do crime.

Se as forças que costuram a conduta criminosa e as respostas estatais ao crime afetam as pessoas de modo desproporcional, acredita-se que uma análise dedicada a compreender os fatores determinantes do controle da criminalidade deve incorporar também a análise de fatores estruturais e exógenos ao sujeito que comete o crime. Isso torna necessária uma interdisciplinaridade capaz de englobar distintos fatores sociais, o que o artigo em questão pretende contribuir ao tecer aspectos da criminalidade à luz distintos arcabouços teóricos e ao incluir na discussão, em termos de contribuições empíricas, os efeitos de elementos socioeconômicos sobre os índices criminais.

Para explicar o comportamento criminoso, um dos contrapontos à linearidade da escolha racional é a abordagem de cunho sociológico que aqui se apresenta por intermédio das seguintes teorias: Teoria da Associação Diferencial, Teoria do Controle Social e Teoria da Desorganização Social. 
A Teoria da Associação Diferencial (Sutherland, 1973) defende que o comportamento criminoso tem origem no processo de socialização do indivíduo e do estabelecimento de suas interações sociais. Essa perspectiva conduz ao entendimento de que assim como os indivíduos aprendem valores, atitudes, técnicas, eles também aprendem o cometimento de crimes e o comportamento não delituoso (Costa \& Varalli, 2018; Dobrow, 2015). Embora a teoria contemple aspectos de nível macro (do ambiente, para além do nível individual/micro) na medida em que enfatiza processos sociológicos e de aprendizagem (assim como racionalizado, o comportamento criminoso é aprendido), parece falhar em explicar como o aprendizado ocorreria na conduta criminosa (Shon \& Barton-Belessa, 2015). Sua contribuição principal está em permitir a reflexão sobre a complexidade que envolve diferentes tipos de crime e de que a transmissão do aprendizado sobre o crime está atrelada às relações interpessoais dos indivíduos (Costa \& Varalli, 2018).

A Teoria do Controle Social (Agnew, 1991), por sua vez, busca explicar em determinados aspectos do meio social, os motivos que levam o indivíduo a cometer (ou não) atos criminosos, como as relações familiares e a realização de atividades educacionais (Lima et al., 2017). Os pressupostos dessa teoria enfatizam a compreensão do porquê os indivíduos se mantêm em conformidade com as normas sociais. Em essência, argumenta-se que um crime pode ocorrer se a solidariedade social não for mantida. Neste sentido, essa perspectiva ressalta que o conceito de self primário é construído pelos indivíduos por meio de associações próximas e frequentes com pessoas do meio em que se inserem. A conformidade pode se manifestar pela aceitação de regras e regulamentos, pelos controles familiares e por técnicas de neutralização utilizadas para justificar comportamentos, por exemplo. Sob essa ótica, comunidades com laços mais distantes estariam sujeitas a níveis mais elevados de criminalidade (Shon \& Barton-Belessa, 2015).

A última teoria a ser explicitada é a Teoria da Desorganização Social (Shaw \& McKay, 1942). Mediante uma visão sistêmica, essa teoria permite a compreensão de que a (des)organização social pode maximizar ou atenuar o controle social e, consequentemente, a prática de crimes, considerando, neste aspecto, elementos como o status socioeconômico, (des)emprego, (des)agregação familiar etc. (Cerqueira \& Lobão, 2004; Mattos, 2018). Embora o foco de argumentação da teoria seja de que há uma concentração geográfica de crimes em determinados locais e bairros (distribuição do crime) e de que as escolhas comportamentais à prática de crime estariam relacionadas ao ambiente físico (geográfico) e social dos indivíduos, essa perspectiva auxilia o entendimento de que o crime pode ser uma resposta ou reação às condições de vida desfavoráveis (Bond, 2015). Nesse sentido, complementa Derziotis (2017) que, sob a ótica dessa teoria, os altos índices criminais em determinados locais devem-se não restritamente a sua localização geográfica, mas também devido a sua associação com questões sociais relevantes, como desemprego, pobreza, baixa renda econômica e outras.

Pode-se resumir até o momento que a primeira abordagem teórica descrita, a Teoria Econômica da Escolha Racional, conduz o entendimento de que o crime é uma ação adotada pelo indivíduo que o pratica, cuja propensão é decidida racionalmente por ele ao ponderar os benefícios (ou recompensas do ato) e os custos (ou riscos da sua ação). Sem detalhar as limitações invocadas pela literatura, a Escolha Racional subsidia a compreensão de uma certa linearidade do crime, quer dizer, os crimes tendem a seguir uma lógica direcionada, considerando o balanceamento realizado pelos sujeitos. Por outro lado, em comum, as teorias sociológicas do crime - evidenciadas nesse capítulo não com a intenção de contrapor à primeira teoria apresentada - problematizam o fenômeno do crime a partir das circunstâncias sociais que tangenciam a sua prática, sejam elas fundadas nas relações interpessoais 
dos indivíduos, no controle social familiar e/ou educacional, na situação socioeconômica das pessoas, e outros elementos.

Em se tratando da produção acadêmica dedicada a investigar fatores determinantes da criminalidade, pautadas nas abordagens teóricas explicitadas, pode-se mencionar na literatura internacional e nacional algumas pesquisas realizadas, as quais são apresentadas a seguir.

Levitt $(1997,2004)$ desenvolveu alguns trabalhos no sentido de investigar fatores responsáveis pela redução de crimes nos Estados Unidos na década de 1990. O economista compreendeu que quatro fatores explicariam a redução do crime no país, sendo eles: o aumento do número de policiais, o crescimento da população carcerária, o recuo da epidemia do crack e a legalização do aborto. Bradford (2011), pesquisador londrino, por seu turno, em contraposição à Levitt, sustentou que o efeito causal do tamanho da força policial sobre a redução dos crimes violentos é fraco e, se considerado o quantitativo de policiais e os crimes contra o patrimônio, a relação tem impacto ínfimo.

Especialista em Criminologia nos Estados Unidos, Stemen (2007), em uma análise do período compreendido entre 1970 e 2005, produziu estudos sobre o impacto do encarceramento sobre o crime no país. O autor confirmou que as pesquisas mais aprofundadas discorrem que o aumento de $10 \%$ no quantitativo de encarceramento gera uma redução de apenas $4 \%$ nos crimes. Pode-se mencionar outros aspectos contributivos do trabalho de Stemen (2007), como demais fatores que possuem impacto positivo sobre a queda das taxas de crimes: o aumento do número de policiais per capita, a redução dos índices de desemprego e o aumento dos níveis de renda e de educação.

Esses apontamentos de Stemen (2007) sobre a relação de aspectos sociais e os índices de criminalidade também são abordados por outros pesquisadores. O estudo empírico com abordagem quantitativa de Mallubhotla (2013), por exemplo, comprovou o efeito positivo e estatisticamente significante entre emprego e crime, concluindo que o aumento do número de empregos tende a reduzir as taxas de criminalidade. Niknami (2012), também mediante um estudo empírico, encontrou evidências da relação entre os níveis de renda da população e os índices de crimes contra o patrimônio, permitindo-se supor que a desigualdade de renda está fortemente relacionada ao comportamento criminal. Não obstante, Hjalmarsson e Lochner (2012) consideraram que as políticas dedicadas à melhoria da educação têm o potencial de atuar tanto positivamente como estrategicamente na redução de crimes.

Em âmbito nacional, Cerqueira e Moura (2019), por meio do desenvolvimento de pesquisa quantitativa, concluíram que a redução da taxa de desemprego na faixa etária de 15 a 29 anos de idade, como consequência do aumento de empregos, tem o potencial de afastar jovens do sexo masculino do mundo do crime no país. Bartz, Quartieri e Menezes (2018), cujo estudo concentrou-se em dados das regiões administrativas do Rio Grande do Sul, identificaram que variáveis relativas à renda da população influenciam no comportamento dos índices criminais. Esses autores, inclusive, expõem que o aumento de renda da população mais pobre impacta na redução de crimes. Anjos Júnior, Lombardi Filho e Amaral (2018), por sua vez, ao buscarem identificar as determinantes da criminalidade na região Sudeste do país, alcançaram segundo os resultados da pesquisa quantitativa a compreensão de que as taxas de homicídios, especificamente, são influenciadas positivamente pela densidade populacional, o PIB per capita, a taxa de desemprego e a proporção de jovens na população.

Percebe-se, pois, pelos resultados do extrato das pesquisas acadêmicas apresentadas, que há divergências e similaridades entre os estudos, o que torna a temática vinculadas às circunstâncias do crime ainda mais relevante. Nesta esteira, o presente estudo apresenta um modelo teórico proposto 
para o teste das hipóteses teóricas, desenhado a partir das abordagens teóricas explicitadas. A Figura 1 a seguir representa o modelo teórico proposto e as hipóteses da pesquisa - a dimensão diretamente relacionada ao construto "controle da criminalidade" é denominada de "elementos socioeconômicos".

\section{FIGURA 1 MODELO TEÓRICO PROPOSTO E HIPÓTESES DA PESQUISA}

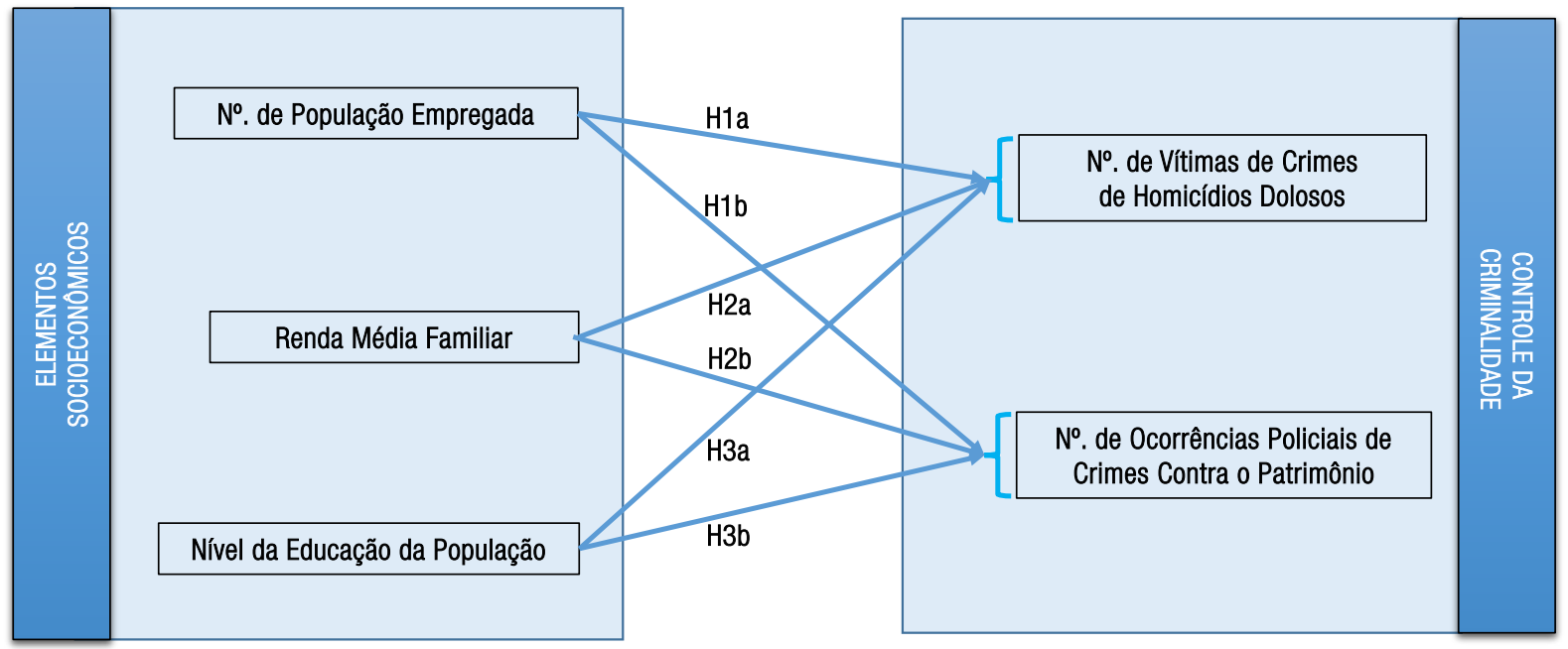

Fonte: Elaborada pelos autores.

O modelo apresenta, de forma específica, as seguintes relações: à esquerda estão as três variáveis que representam os "elementos socioeconômicos", sendo elas 1. no de população empregada; 2 . renda média familiar; e 3. nível de educação da população. Essas três variáveis também são conhecidas como variáveis independentes (VI) já que teoricamente influenciam as variáveis dependentes. À direita está o construto "controle da criminalidade", constituído pelas variáveis dependentes (VD): 1. $\mathrm{n}^{\circ}$ de vítimas de homicídios dolosos e 2. ํㅡㄹ de ocorrências policiais de crimes contra o patrimônio.

O controle dos índices de crimes de homicídio doloso e de crimes contra o patrimônio são considerados os maiores desafios dos gestores da área da segurança pública e são usualmente utilizados nas pesquisas acadêmicas com foco no assunto. Messner (1982), ao estudar as relações entre pobreza, desigualdade e crimes, utilizou como referência para as correlações os crimes de homicídio. Fajnzylber, Lederman e Loayza (2002), por sua vez, ao investigarem a causalidade entre desigualdade de renda e crimes violentos, utilizaram as taxas de homicídios e de roubos. Já Levitt (2004), ao aprofundar a análise sobre as causas do declínio dos crimes nos Estados Unidos, teve como referência as estatísticas de homicídios e de crimes de propriedade (assaltos, latrocínios, furtos). 


\subsection{Elementos socioeconômicos}

A dimensão denominada de "elementos socioeconômicos", compreende variáveis independentes (VI) como o número da população empregada, renda média familiar e nível de educação da população. A Teoria Econômica da Escolha Racional (Becker, 1968; Ehrlich, 1973) abarca a dimensão em destaque tendo em vista que defende a perspectiva de custos-benefícios da conduta dos indivíduos diante da propensão à atividade criminosa (Strapazzon \& Tramontina, 2015). Campos (2008) explica com base nesta abordagem teórica que variáveis como a eficiência do aparato da polícia e do sistema de justiça e a severidade de punições de crimes são fatores que tendem a reduzir a propensão ao crime; e, com efeito semelhante, as variáveis relacionadas à renda, ao salário e à educação são fatores positivos que tendem a afastar o indivíduo da conduta criminosa.

Consistentemente dentro dessa lógica, observa-se que as abordagens sobre o crime de base sociológica complementam a Teoria Econômica da Escolha Racional na tentativa de relacionar aspectos sociais ao controle da criminalidade.

A Teoria da Associação Diferencial (Sutherland, 1973), por exemplo, interessa ao escopo desse estudo ao apontar que a conduta criminosa é resultado de contextos grupais, processos de interação e de comunicação do indivíduo (Cerqueira \& Lobão, 2004; Martins \& Gomes, 2011) e, nesse aspecto, instituições e redes sociais responsáveis pela socialização primária do indivíduo (como a família, escola, vizinhança etc.) têm papel preponderante para o ingresso ou não no mundo do crime (Costa \& Varalli, 2018).

A Teoria do Controle Social (Agnew, 1991), por sua vez, defende que quanto maior o nível de envolvimento do indivíduo com o sistema social, maior é a aliança deste com valores e normas vigentes, o que, consequentemente, o afastaria da prática de crimes (Anjos, Lombardi, Ciríaco \& Silva, 2018; Cerqueira \& Lobão, 2004).

A Teoria da Desorganização Social (Shaw \& McKay, 1942), por seu turno, argumenta que o crime deve ser entendido por meio das características do ambiente social do indivíduo, apontando como aspectos da desorganização social que influenciam a prática de crimes: "famílias desestruturadas, alta mobilidade residencial, reduzidas oportunidades educacionais e no mercado de trabalho" (Viapiana, 2006, p. 162).

Portanto, a partir da referida construção teórica, apresenta-se as hipóteses da pesquisa no que diz respeito aos "elementos socioeconômicos":

- H1a: O aumento do número da população empregada influencia positivamente no controle dos crimes de homicídios dolosos;

- H1b: O aumento do número da população empregada influencia positivamente no controle dos crimes contra o patrimônio;

- H2a: O aumento da renda média familiar influencia positivamente no controle dos crimes de homicídios dolosos;

- H2b: O aumento da renda média familiar influencia positivamente no controle dos crimes contra o patrimônio;

- H3a: O aumento do nível de educação da população influencia positivamente no controle dos crimes de homicídios dolosos; e

- H3b: O aumento do nível de educação da população influencia positivamente no controle dos crimes contra o patrimônio. 


\section{ASPECTOS METODOLÓGICOS}

\subsection{Fonte e coleta de dados}

\subsubsection{Variáveis Independentes}

Os dados coletados que se referem aos "elementos socioeconômicos" são compostos pelas seguintes variáveis: 1. número da população empregada (OCU); 2. renda média familiar (SAL) e; 3. nível de educação da população (ALF). Dessa forma, tendo como referência as bases nacionais disponíveis que envolvem as variáveis propostas para medir tal construto, definiu-se como proxies:

OCU: Taxa da população ocupada;

SAL: Taxa de salários e outras remunerações dos trabalhadores formais; e,

ALF: Taxa de alfabetização da população.

A OCU foi calculada da seguinte forma: número da população ocupada em determinada área geográfica dividido pelo total da população da área geográfica e, posteriormente, multiplicado por 100. A fonte dos dados da população ocupada provém do Cadastro Central de Empresas (CEMPRE) do Instituto Brasileiro de Geografia e Estatística (IBGE), cujos dados foram extraídos do site do órgão estatal (acesso aberto ou livre).

A variável relativa à renda média familiar, por sua vez, foi traduzida na proxy "taxa de salários e outras remunerações dos trabalhadores formais" (SAL). A opção por esta proxy se deu pela disponibilização facilitada do acesso aos dados por meio do site do órgão estatal (CEMPRE/IBGE) e pela periodicidade anual de divulgação dos dados, em detrimento dos dados de renda média familiar, cuja divulgação de cada município ocorre concomitantemente aos Censos Demográficos do IBGE. A proxy foi calculada da respectiva forma: salários e outras remunerações (tabuladas em mil reais) em determinada área geográfica, dividido pelo total da população da área geográfica e, posteriormente, multiplicado por 100.

Por último, a "taxa de alfabetização da população" (ALF) foi extraída de maneira fidedigna a partir do acesso ao Sistema IBGE de Recuperação Automática (SIDRA).

As taxas da população ocupada (OCU) e de salários e outras remunerações (SAL) foram calculadas para cada um dos 78 (setenta e oito) municípios do estado do Espírito Santo, distribuídos para os anos de 2001 a 2015. A fonte dos dados populacionais dos municípios do estado também é do IBGE. E em se tratando da taxa de alfabetização da população, os dados municipais disponíveis referem-se aos Censos Demográficos (IBGE) dos anos de 2000 e 2010, logo, para o banco de dados formulado para esta pesquisa, considerou-se do ano de 2001 a 2009, os dados do Censo de 2000 e, dos anos de 2010 a 2015, utilizaram-se os dados do Censo de 2010.

\subsubsection{Variáveis Dependentes}

As variáveis utilizadas na pesquisa para medir a dimensão "controle da criminalidade" foram: 1. no de vítimas de homicídios dolosos e 2. no de ocorrências policiais de crimes contra o patrimônio. Considerando a realização de trabalhos acadêmicos semelhantes ao objeto de estudo proposto, as variáveis indicadas foram traduzidas nas seguintes proxies: 
HOM: Taxa de homicídios dolosos para cada 100 mil habitantes; e

PAT: Taxa de crimes contra o patrimônio para cada 100 mil habitantes.

A "taxa de homicídios dolosos para cada 100 mil habitantes" (HOM) foi calculada por meio da seguinte sistemática: número de vítimas de homicídios dolosos (crime praticado intencionalmente) em determinada área geográfica, dividido pelo total da população da área geográfica, e, posteriormente, multiplicado por 100 mil (Secretaria da Segurança Pública e Administração Penitenciária, 2016).

No que se refere à "taxa de crimes contra o patrimônio para cada 100 mil habitantes" (PAT), optou-se por selecionar os tipos de crimes contra o patrimônio com maior incidência no Espírito Santo (critério de seleção) no período de 2006 a 2015. Sendo eles: 1. roubo a pessoa; 2 . roubo em transporte coletivo; 3. roubo em comércio; 4. roubo em residência e; 5. furto e roubo de veículo. A partir do acesso à base de dados de ocorrências registradas desses crimes, procedeu-se à somatória do quantitativo das ocorrências para a consolidação do "número de ocorrências de crimes contra o patrimônio". Essa taxa foi calculada da seguinte forma: número de ocorrências de crimes contra o patrimônio em determinada área geográfica dividido pelo total da população da área geográfica e, posteriormente, multiplicado por 100 mil (Secretaria da Segurança Pública e Administração Penitenciária, 2016). As taxas de homicídios dolosos e de crimes contra o patrimônio foram calculadas para cada um dos 78 (setenta e oito) municípios do Espírito Santo.

Os dados relativos ao quantitativo de vítimas de homicídios dolosos e de ocorrências de crimes contra o patrimônio têm como fonte os Boletins de Ocorrência Policial (BOs), cuja base de dados é gerenciada e disponibilizada pela Secretaria de Estado da Segurança Pública e Defesa Social do Espírito Santo (SESP).

Os dados populacionais dos municípios do estado, necessários para o cálculo das taxas, tiveram como fonte o IBGE, que fornece anualmente as estimativas populacionais dos municípios e dos estados. Registra-se ainda que, conforme disponibilidade dos dados da SESP, as taxas foram calculadas para os anos de 2001 a 2015 no caso dos homicídios dolosos e, para os anos de 2006 a 2015, os crimes contra o patrimônio.

\subsection{Técnica de análise dos dados}

Diante da apresentação da proposta do modelo teórico, das hipóteses do estudo e das variáveis explicitadas (proxies) e, visando alcançar resposta ao problema de pesquisa, propõe-se como estratégia de análise dos dados o modelo de regressão linear por meio do tratamento estatístico de dados com auxílio do software SPSS Statistics, versão 25.

De acordo com Field (2009), a essência da análise de regressão está na possibilidade de prever um resultado (variável dependente), a partir de uma ou mais variáveis previsoras (variáveis independentes). Ou seja, a análise de regressão é o método que tem como objetivo medir a mudança média da variável dependente que é associada a uma ou mais variáveis independentes. Neste presente estudo, propõe-se duas análises de regressão linear: a primeira (Teste 1), tendo como variável dependente a taxa de homicídios dolosos (HOM) e; a segunda (Teste 2), tendo como variável dependente a taxa de crimes contra o patrimônio (PAT). Portanto, foram testadas as seguintes hipóteses: H1a, H1b, H2a, H2b, H3a e H3b, cujos resultados são descritos na próxima seção. 


\section{APRESENTAÇÃO DOS DADOS E DISCUSSÃO DOS RESULTADOS}

Os principais aspectos das estatísticas descritivas do Teste 1, realizado a partir da variável dependente taxa de homicídios dolosos para cada 100 mil habitantes (HOM) e das três variáveis independentes taxa da população ocupada (OCU), taxa de salários e outras remunerações dos trabalhadores formais (SAL) e taxa de alfabetização da população (ALF), podem ser observados na Tabela 1, que contempla o resumo do modelo, o teste ANOVA, os coeficientes e a correlação (resultados extraídos do software SPSS).

\section{TABELA 1 ESTATÍSTICAS DESCRITIVAS DO TESTE 1}

\begin{tabular}{|c|c|c|c|c|c|c|}
\hline \multicolumn{7}{|c|}{ RESUMO DO MODELO } \\
\hline Modelo & $\mathbf{R}$ & \multicolumn{2}{|c|}{ R quadrado } & $\mathrm{R}$ quadrado ajustado & \multicolumn{2}{|c|}{ Erro-padrão da estimativa } \\
\hline 1 & $0,176^{\mathrm{a}}$ & \multicolumn{2}{|c|}{0,031} & 0,028 & \multicolumn{2}{|c|}{24,98786877} \\
\hline \multicolumn{7}{|c|}{ ANOVA $^{\mathrm{a}}$} \\
\hline \multicolumn{2}{|l|}{ Modelo } & $\begin{array}{l}\text { Soma dos } \\
\text { Quadrados }\end{array}$ & df & Quadrado Médio & Z & Sig. \\
\hline \multirow{3}{*}{1} & Regressão & 23178,986 & 3 & 7726,329 & \multirow[t]{3}{*}{12,374} & \multirow[t]{3}{*}{0,000} \\
\hline & Resíduo & 728042,921 & 1166 & 624,394 & & \\
\hline & Total & 751221,907 & 1169 & & & \\
\hline \multicolumn{7}{|c|}{ COEFICIENTES } \\
\hline \multirow[t]{2}{*}{ Modelo } & & \multicolumn{2}{|c|}{ Coeficientes não padronizados } & $\begin{array}{c}\text { Coeficientes } \\
\text { padronizados }\end{array}$ & \multirow[t]{2}{*}{$\mathrm{T}$} & \multirow[t]{2}{*}{ Sig } \\
\hline & & B & Erro Padrão & Beta & & \\
\hline \multirow{4}{*}{1} & (Constante) & 16,497 & 7,304 & & 2,259 & 0,024 \\
\hline & OCU & 0,472 & 0,120 & 0,195 & 3,932 & 0,000 \\
\hline & $\mathrm{SAL}$ & $-0,004$ & 0,005 & $-0,39$ & $-0,803$ & 0,422 \\
\hline & ALF & 0,070 & 0,090 & 0,024 & 0,774 & 0,439 \\
\hline \multicolumn{7}{|c|}{ CORRELAÇÃO } \\
\hline & & HOM & OCU & SAL & \multicolumn{2}{|c|}{ ALF } \\
\hline \multirow{4}{*}{$\begin{array}{l}\text { Correlação de } \\
\text { Pearson }\end{array}$} & HOM & 1,000 & 0,173 & 0,125 & \multicolumn{2}{|c|}{0,085} \\
\hline & OCU & 0,173 & 1,000 & 0,803 & \multicolumn{2}{|c|}{0,379} \\
\hline & SAL & 0,125 & 0,803 & 1,000 & \multicolumn{2}{|c|}{0,319} \\
\hline & ALF & 0,085 & 0,379 & 0,319 & \multicolumn{2}{|c|}{1,000} \\
\hline
\end{tabular}

Fonte: Elaborada pelos autores. 
O modelo de regressão linear do Teste 1 é apresentado pela respectiva fórmula: $\mathrm{HOM}=\mathrm{b} 1 \mathrm{OCU}$ + b2SAL + b3ALF + a. A avaliação dos resultados provenientes do referido teste permite discutir alguns achados.

Inicialmente, observa-se que o coeficiente de correlação R, que indica o grau de associação entre a taxa de homicídios dolosos (VD) e o conjunto de variáveis independentes (ocupação da população, salários e alfabetização) é de 0,176 . Com isso é possível inferir que o conjunto de fatores determinantes pouco influência nos homicídios.

O coeficiente de determinação da variância $\mathrm{R}^{2}$, por sua vez, corrobora a conclusão sobre a "fraca" explicação do modelo, considerando seu resultado de 0,031. Dessa maneira, é possível inferir que existe baixa correlação entre todas as VI's com a VD.

Os coeficientes também demonstraram que os pesos não padronizados (B) e os pesos padronizados (Beta) são positivos e consideráveis com relação à variável independente OCU (ocupação); por outro lado, os resultados são negativos com relação à SAL (salários) e pouco expressivos com relação à ALF (alfabetização).

Por fim, o teste de correlação de Pearson demonstrou que a variável dependente HOM possui maior correlação positiva com a variável independente OCU (ocupação). Interessante destacar que não houve ocorrência de correlação negativa dos homicídios com qualquer variável independente. Além disso, identificou-se considerável grau de correlação entre OCU (ocupação) e SAL (salários) e, em menor proporção, mas também de forma positiva, com a ALF (alfabetização).

Na sequência, os elementos de destaque das estatísticas descritivas do Teste 2, realizado a partir da variável dependente taxa de crimes contra o patrimônio para cada 100 mil habitantes (PAT) e as três variáveis independentes OCU, SAL e ALF podem ser observados na Tabela 2.

\section{TABELA 2 ESTATÍSTICAS DESCRITIVAS DO TESTE 2}

\begin{tabular}{|c|c|c|c|c|c|c|}
\hline \multicolumn{7}{|c|}{ RESUMO DO MODELO } \\
\hline Modelo & $\mathrm{R}$ & & $\mathrm{R}$ quadrado & $\begin{array}{l}\text { R quadrado } \\
\text { ajustado }\end{array}$ & \multicolumn{2}{|c|}{ Erro-padrão da estimativa } \\
\hline 1 & $0,534^{a}$ & & 0,285 & 0,282 & \multicolumn{2}{|c|}{175,8189799} \\
\hline \multicolumn{7}{|c|}{ ANOVA $^{\mathrm{a}}$} \\
\hline Modelo & & Soma dos Quadrados & Df & $\begin{array}{c}\text { Quadrado } \\
\text { Médio }\end{array}$ & Z & Sig \\
\hline \multirow{3}{*}{1} & Regressão & 9553752,222 & 3 & 3184584,074 & \multirow[t]{3}{*}{103,020} & \multirow[t]{3}{*}{0,000} \\
\hline & Resíduo & 23987955,42 & 776 & 30912,314 & & \\
\hline & Total & 33541707,64 & 779 & & & \\
\hline
\end{tabular}




\begin{tabular}{|c|c|c|c|c|c|c|}
\hline \multicolumn{7}{|c|}{ COEFICIENTES } \\
\hline \multirow{2}{*}{ Modelo } & & \multicolumn{2}{|c|}{ Coeficientes não padronizados } & Coeficientes & \multirow{2}{*}{$\mathrm{T}$} & \multirow{2}{*}{ Sig } \\
\hline & & B & Erro Padrão & Beta & & \\
\hline \multirow{4}{*}{1} & (Constante) & $-291,961$ & 73,333 & & $-3,981$ & 0,000 \\
\hline & $\mathrm{OCU}$ & 4,923 & 1,185 & 0,245 & 4,156 & 0,000 \\
\hline & SAL & 0,164 & 0,042 & 0,225 & 3,910 & 0,000 \\
\hline & ALF & 4,319 & 0,898 & 0,159 & 4,813 & 0,000 \\
\hline \multicolumn{7}{|c|}{ CORRELAÇÃO } \\
\hline & & PAT & OCU & SAL & \multicolumn{2}{|c|}{ ALF } \\
\hline \multirow{4}{*}{$\begin{array}{l}\text { Correlação de } \\
\text { Pearson }\end{array}$} & PAT & 1,000 & 0,500 & 0,486 & \multicolumn{2}{|c|}{0,332} \\
\hline & OCU & 0,500 & 1,000 & 0,849 & \multicolumn{2}{|c|}{0,398} \\
\hline & SAL & 0,486 & 0,849 & 1,000 & \multicolumn{2}{|c|}{0,334} \\
\hline & ALF & 0,332 & 0,398 & 0,334 & \multicolumn{2}{|c|}{1,000} \\
\hline
\end{tabular}

Fonte: Elaborada pelos autores.

O modelo de regressão linear do Teste 2 é representado pela seguinte fórmula: $\mathrm{PAT}=\mathrm{b} 1 \mathrm{OCU}+$ b2SAL + b3ALF + a. Semelhantemente à análise empreendida para o Teste 1, é possível tecer alguns comentários com base nos resultados extraídos para o Teste 2 .

Verifica-se que o coeficiente de correlação R $(0,534)$ é maior do que ao identificado no Teste 1 (homicídios), o que denota que o conjunto de fatores determinantes (ocupação, salários e alfabetização) influenciam mais os crimes contra o patrimônio (PAT) do que os homicídios dolosos (HOM).

Já o resultado do coeficiente de determinação da variância $R^{2}(0,285)$ também é maior do que o observado no Teste $1\left(R^{2}=0,031\right)$, assim, pode-se considerar que o modelo do Teste 2 explica melhor a relação das variáveis independentes com a variável dependente PAT. Isto significa que as variáveis independentes (OCU, SAL e ALF) se constituem em bons preditores para explicar a variação que acontece no comportamento da variável consequente, crimes contra o patrimônio.

Por conseguinte, os coeficientes demonstraram que os pesos não padronizados (B) e os pesos padronizados (Beta) são positivos e consideráveis com relação à variável independente OCU (ocupação) e ALF (alfabetização). Já a correlação de Pearson demonstrou que a variável PAT mantém um grau de correlação positiva mais intensa com OCU (ocupação) e SAL (salários) - apresentando, inclusive, valores bem próximos.

Ainda, como no Teste 1, não houve correlação negativa dos crimes contra o patrimônio com qualquer variável independente. A correlação mais "fraca" encontrada foi entre a variável PAT com a ALF (alfabetização). Destaca-se, além disso, o considerável grau de correlação da OCU (ocupação) com SAL (salários) e, em menor proporção, mas também de maneira positiva, com ALF, como também pode ser verificado no Teste 1 .

No geral, o modelo evidencia algumas relações robustas entre a VD (crimes contra o patrimônio) e as VI's, bem como relações entre as próprias variáveis independentes. Contudo, vale salientar que apesar das varáveis dependentes HOM e PAT apresentarem índices $\mathrm{R}^{2}$ baixos, tal fator não invalida 
os resultados encontrados de associações positivas e significantes entre as variáveis que conformam o modelo de pesquisa. Nesse sentido, Hair, Hult e Sarstedt (2014) afirmam que o valor de $\mathrm{R}^{2}$ em níveis elevados indicam maiores níveis de precisão, no entanto, em que pese tal constatação, os autores mencionam que não existem regras práticas para estabelecimento de valores aceitáveis/razoáveis de $\mathrm{R}^{2}$ e, que deve-se levar em consideração fatores como a complexidade do modelo, da disciplina de pesquisa ou da área de conhecimento dedicada ao estudo. Assim, segundo os autores, valores de $\mathrm{R}^{2} \mathrm{de}$ até 0,20 são considerados altos em disciplinas que estudam comportamento (Hair et al., 2014, p. 175), como no presente estudo, que de alguma maneira tangencia aspectos comportamentais da segurança pública. Ademais, nas Ciências Sociais Aplicadas, na qual se insere a pesquisa, a relação entre variáveis já é considerada como um cerne dos testes de interações (Cohen, Cohen, West \& Aiken, 2003).

Assim, retomando às proposições teóricas que foram factíveis de serem testadas nesse estudo, é apresentado no Quadro 1 um breve resumo dos resultados obtidos com o teste das hipóteses, a partir da observância dos níveis de significância das correlações.

\section{QUADRO 1 RESULTADOS CONSOLIDADOS DO TESTE DE HIPÓTESES}

\begin{tabular}{|c|c|}
\hline HIPÓTESE TEÓRICA & RESULTADO \\
\hline $\begin{array}{l}\text { H1a: } 0 \text { aumento do número da população empregada influencia positivamente no controle dos } \\
\text { crimes de homicídios dolosos. }\end{array}$ & Suportada \\
\hline $\begin{array}{l}\text { H1b: } 0 \text { aumento do número da população empregada influencia positivamente no controle dos } \\
\text { crimes contra o patrimônio. }\end{array}$ & Suportada \\
\hline $\begin{array}{l}\text { H2a: } 0 \text { aumento da renda média familiar influencia positivamente no controle dos crimes de } \\
\text { homicídios dolosos. }\end{array}$ & Rejeitada \\
\hline $\begin{array}{l}\text { H2b: } 0 \text { aumento da renda média familiar influencia positivamente no controle dos crimes contra } 0 \\
\text { patrimônio. }\end{array}$ & Suportada \\
\hline $\begin{array}{l}\text { H3a: } 0 \text { aumento do nível de educação da população influencia positivamente no controle dos } \\
\text { crimes de homicídios dolosos. }\end{array}$ & Rejeitada \\
\hline $\begin{array}{l}\text { H3b: } 0 \text { aumento do nível de educação da população influencia positivamente no controle dos } \\
\text { crimes contra o patrimônio. }\end{array}$ & Suportada \\
\hline
\end{tabular}

Fonte: Elaborado pelos autores.

Ao se discutir os achados desse estudo à luz das referências teóricas, observa-se que os resultados demostraram congruência com as considerações da abordagem relacionada à Teoria Econômica da Escolha Racional (Becker, 1968; Ehrlich, 1973), uma vez que a análise demonstrou que os crimes de homicídios e contra o patrimônio possuem correlação com a dimensão socioeconômica referente à ocupação (empregabilidade) da população. Diante da escolha racional de cometer crimes, a variável referente à ocupação da população (emprego) se torna um fator positivo ao indivíduo (de caráter preventivo) e desestimulante à prática de crimes, conforme defendido por Scheeffer (2013), Cerqueira e Lobão (2004) e Strapazzon e Tramontina (2015).É possível supor ainda, que quanto mais 
oportunidades de emprego houver para a população, maior será a probabilidade de controle sobre os crimes de homicídios dolosos e crimes contra o patrimônio.

Convém ressaltar, a este respeito, que o coeficiente de correlação $\mathrm{R}$ do Teste 2 (crimes contra patrimônio), sendo maior do que aquele identificado no Teste 1 (homicídios), traduzido em uma maior influência dos fatores "ocupação, salário e alfabetização" sobre crimes contra o patrimônio (PAT) do que sobre homicídios dolosos (HOM), reitera a crítica de Loughran et al. (2016) de que a Teoria da Escolha Racional, em contexto aplicável ao crime, limitar-se-ia a explicar crimes contra a propriedade, mostrando-se insuficiente, por si só, para explicar na prática crimes violentos, como os homicídios.

No caso do teste do modelo de regressão realizado com a variável dependente crimes contra o patrimônio (Teste 2), verifica-se que, além da influência positiva relacionada ao "emprego", a variável independente "salários" (ou renda) recebeu destaque. Isso potencializa a capilaridade desta correlação do modelo com a Teoria Econômica da Escolha Racional, com as Teorias do Controle Social e da Desorganização Social. Pode-se considerar que indivíduos empregados e com renda (trabalhos formais) são menos propensos à prática de crimes contra o patrimônio. A Teoria do Controle Social auxilia nesse entendimento porque explica que quanto maior o envolvimento de indivíduos com o sistema social - emprego e renda devido ao trabalho formal e com atividades na área da educação -, maior é a ligação destes com valores e normas vigentes, distanciando-os da prática de crimes (Anjos et al., 2018; Cerqueira \& Lobão, 2004).

Seguindo o mesmo raciocínio, tem-se que as condições de organização social, como a inserção e atuação no mercado de trabalho, afastam o indivíduo da conduta de práticas criminosas, entendimento este consubstanciado pela Teoria da Desorganização Social (Shaw \& McKay, 1942). Dessa forma, as análises deste estudo convergem com os resultados obtidos em outras pesquisas acadêmicas realizadas com foco nesse fenômeno, como os trabalhos de Stemen (2007), Niknami (2012), Mallubhotla (2013) e Cerqueira e Moura (2019).

\section{CONCLUSÕES}

Com a finalidade de identificar os fatores determinantes para o controle dos índices de criminalidade a partir de uma amostra do estado do Espírito Santo, os resultados deste esforço de pesquisa apresentam achados relevantes tanto do ponto de vista prático (gerencial e social), quanto de sua relevância acadêmica.

A pesquisa apresentou um modelo teórico alicerçado em duas abordagens teóricas - a Teoria Econômica da Escolha Racional e o conjunto de teorias sociológicas composto pelas Teorias da Associação Diferencial, do Controle Social e da Desorganização Social -, tendo como foco de investigação a influência de elementos socioeconômicos sobre a variável dependente "controle da criminalidade".

O estudo de caráter quantitativo utilizou-se da coleta de dados secundários, obtidos por meio de acesso a bases de dados de órgãos públicos estaduais e federais. Não se pode olvidar que ocorreu limitação na coleta dos dados, sendo possível até o presente momento a obtenção de dados referentes apenas a homicídios dolosos e a crimes contra o patrimônio (variáveis dependentes). E, em relação às variáveis independentes, ao número da população empregada, aos salários e a outras remunerações dos trabalhadores formais e, ao nível de alfabetização da população (variáveis independentes da dimensão 
"elementos socioeconômicos"). Apesar das limitações, tais fatores não desqualificam a pesquisa que, ao seguir as recomendações da literatura consultada, cumpriu com rigor as etapas metodológicas, respondeu à problemática invocada no estudo, bem como atendeu ao objetivo a que se propôs.

Diante das considerações realizadas e das abordagens teóricas evidenciadas no conteúdo textual do artigo, é possível afirmar, em um primeiro momento, que a confirmação das hipóteses H1a e H1b encontra respaldo nas teorias: Econômica da Escolha Racional, Controle Social e Desorganização Social. A pesquisa ratificou a argumentação de que os níveis de empregabilidade têm potencial de atuar positivamente sobre o controle da criminalidade (crimes de homicídios dolosos e crimes contra o patrimônio).

Em segundo lugar, a confirmação da hipótese $\mathrm{H} 2 \mathrm{~b}$, no que diz respeito a influência positiva da renda média familiar sobre o controle dos crimes contra o patrimônio, também é coerente com as discussões das teorias supracitadas. Questões relacionadas à renda da população e às interações dos indivíduos com atividades laborais e mercado de trabalho formal tendem a influenciar os índices de crimes contra o patrimônio. De fato, os elementos sociais relativos a emprego e renda são pontos cruciais para a prevenção de crimes de natureza patrimonial. E, em terceiro lugar, suspeita-se que as possíveis relações entre o nível de educação da população, o processo de socialização do indivíduo e a propensão à prática de crimes patrimoniais podem ser mais bem explicadas à luz das Teorias da Associação Diferencial e do Controle Social.

Portanto, os resultados da pesquisa permitem constatar que os fatores determinantes para o controle dos índices de criminalidade no estado do Espírito Santo envolvem, principalmente, aspectos relacionados à empregabilidade da população.

Isto posto, em termos teóricos, vislumbrou-se a potencialidade do estudo de, por intermédio da integração das distintas abordagens teóricas adotadas, contribuir para a compreensão de que a escolha pela prática de crimes encontra justificativas no benefício que se pode extrair do ato, conjugado a influências do meio social e do contexto socioeconômico a que estão submetidos os indivíduos. Pode-se dizer que a presente pesquisa conseguiu harmonizar as teorias mencionadas, convergindo-as em um ponto comum e sólido: fatores relacionados à empregabilidade da população ajudam a compreender o fenômeno estudado, qual seja, o controle da criminalidade.

Em termos práticos, por sua vez, essas constatações provenientes do estudo podem gerar implicações para uma melhor gestão de organizações de segurança pública brasileiras e respaldar argumentações técnicas de gestores públicos no tocante à necessidade de direcionamento de recursos públicos para a execução de políticas e ações sociais efetivas que potencialmente tendem a impactar, a longo prazo, o cenário da segurança pública. Ressalta-se que políticas multidisciplinares e transversais que conjuguem ações de natureza policial e ações sociais, realizadas de maneira integrada, podem influenciar positivamente a segurança pública.

No caso desse estudo, ficou claro que as ações sociais devem ser direcionadas à geração de emprego e renda à população, o que pode incluir a oferta de cursos de qualificação profissional, incentivos fiscais para a atração e a manutenção de empresas, disponibilização de linhas de crédito para micro e pequenas empresas, por exemplo. Cabe destacar que essas considerações valem para o estado do Espírito Santo (foco do estudo) e para outros estados que possuem características contextuais e sociais semelhantes.

Adicionalmente, vale mencionar que o desenvolvimento e os resultados práticos de pesquisas acadêmicas como esta propiciam o estreitamento das interações entre a academia e a gestão pública, 
proporcionando respostas seguras diante de questões sociais, bem como embasam o fomento, a construção e a consolidação de agenda de pesquisas neste campo.

Como possibilidade de futuras investigações, recomenda-se com o intuito de preencher lacunas da Teoria Econômica da Escolha Racional na Criminologia - particularmente, no que diz respeito à extensão em que as percepções de riscos e recompensas são condicionadas por diferenças entre os indivíduos -, o desenvolvimento de pesquisas que incluam o efeito moderador de fatores psicológicos na percepção de riscos e recompensas em condutas criminais, incluindo nos testes variáveis de controle, como características geográficas e demográficas, etnia, idade e gênero, além daquelas relativas à exposição à violência, ao histórico do indivíduo e envolvimento anterior dele e de familiares em crimes.

Por fim, sugere-se, ainda, novos estudos que busquem ampliar o modelo teórico e as hipóteses de pesquisa propostos no presente artigo, contemplando, além da dimensão "elementos socioeconômicos", outras dimensões que supostamente indicam influenciar o controle da criminalidade conforme a literatura, tais como: 1. dimensão "elementos da atividade policial" (variável como $\mathrm{n}^{\circ} \stackrel{\circ}{ }$ de policiais, subdividido pelo quantitativo referente a cada polícia estadual) e 2. dimensão "elementos do sistema de justiça" (variável como $\mathrm{n}^{\circ}$ da população carcerária). Para a coleta dessas variáveis, é necessário o acesso aos dados oficiais desagregados da administração pública estadual. Acrescenta-se que no que concerne à dimensão "elementos socioeconômicos", sugere-se a coleta de dados de outras variáveis, como: índice de desenvolvimento da educação básica, média de anos de estudo, população assalariada, número de empregos criados, renda média domiciliar per capita, taxa de desemprego e outros, com vistas a se alargar o entendimento acerca do fenômeno estudado dentro do campo de pesquisa em gestão de políticas de segurança pública. 


\section{REFERÊNCIAS}

Afonso, J. R. (2017). Gastos públicos com segurança pública. Revista Conjuntura Econômica, 71(11), 26-28.

Agnew, R. (1991). A longitudinal test of social control theory and delinquency. Journal of Research in Crime and Delinquency, 28, 126-156.

Alessi, G. (2018, novembro 01). Os planos de Bolsonaro para ampliar o porte de arma e a legítima defesa. El País. Recuperado de https://brasil.elpais.com/ brasil/2018/10/30/politica/1540926432_647859.html

Anjos, O. R., Jr., Lombardi, S. C., Filho, \& Amaral, P. V. M. (2018). Determinantes da criminalidade na região sudeste do Brasil: uma aplicação de painel espacial. Economía, sociedad y territorio, 18(57), 525-556.

Anjos, O. R., Jr., Lombardi, S. C., Filho, Ciríaco, J. S., \& Silva, M. V. B. (2018). Testando a hipótese de dependência espacial na taxa de crime dos municípios da região sul do Brasil. Desenvolvimento Regional em Debate, 8(1), 118-141.

Barbosa, R. (2017, junho 20). Fim do regime semiaberto: menos violência ou retrocesso? Gazeta do Povo. Recuperado de https://www. gazetadopovo.com.br/justica/fim-do-regimesemiaberto-menos-violencia-ou-retrocesso8vhtxcu5qsc3pm0ibwkxxahnl/

Bartz, M., Quartieri, E., \& Menezes, G. (2018). Criminalidade no Rio Grande do Sul: uma análise econométrica para os COREDES no ano de 2010. Revista Brasileira de Estudos Regionais e Urbanos, 12(1), 110-128.

Becker, G. (1968). Crime and punishment: an economic approach. Journal of Political Economy, $76,169-217$.

Bond, M. (2015, março 11). Criminology: Social Disorganization Theory Explained. E-Roll Call Magazine. Recuperado de https:// andragogytheory.com/2015/03/11/criminologysocial-disorganization-theory-explained/

Bradford, B. (2011). Police numbers and crime rates: a rapid evidence review. London, UK: Her Majesty's Inspectorate of Constabulary.

Campos, M. S. (2008). Escolha racional e criminalidade: uma avaliação crítica do modelo. Revista SJRJ, 22, 93-110.
Cerqueira, D. (2014). Causas e consequências do crime no Brasil. Rio de Janeiro, RJ: BNDES.

Cerqueira, D., \& Lobão, W. (2004). Determinantes da criminalidade: arcabouços teóricos e resultados empíricos. Revista de Ciências Sociais, 47(2), 233269.

Cerqueira, D., \& Moura, R. (2019). Oportunidades laborais, educacionais e homicídios no Brasil. Brasília, DF: Ipea.

Cohen, J., Cohen, P., West, S. G., \& Aiken, L. S. (2003). Applied multiple regression/correlation analysis for the behavioral sciences. London, UK: Lawrence Erlbaum Associates Publishers.

Constituição [da] República Federativa do Brasil. (1988). Brasília, DF: Senado Federal.

Costa, L. S., \& Varalli, J. T. D. (2018). A Teoria da Associação Diferencial e seus aspectos inseridos na formação do Primeiro Comando da Capital. Revista de Criminologias e Políticas Criminais, 4(2), 159-173.

Derziotis, I. (2017). Critically examine the idea that neighbourhoods characterized by higher levels of 'social disorganization' are more prone to crime. Journal of Forensic Sciences and Criminal Investigation, 1(4), 1-5.

Dobrow, J. A. (2015). Differential Association Theory. In: Jennings, W. G. (Ed.). The Encyclopedia of Crime \& Punishment. Nova Jersey, NJ: WileyBlackwell.

Ehrlich, I. (1973). Participation in illegitimate activities: a theoretical and empirical investigation. Journal of Political Economy, 81, 521-565.

Fajnzylber, P., Lederman, D., \& Loayza, N. (2002). Desigualdade e crime violento. Revista de Direito e Economia, 45(1), 1-40.

Field, A. (2009). Descobrindo a estatística usando o SPSS. Porto Alegre, RS: Artmed.

Fórum Brasileiro de Segurança Pública. (2018). Anuário Brasileiro de Segurança Pública 2018. São Paulo, SP: FBSP.

Hair Jr, J. F., Hult, G. T. M. \& Sarstedt, M. (2014). A primer on partial least squares structural equation modeling (PSL-SEM). London, UK: Sage Publications.

Hjalmarsson, R. \& Lochner, L. (2012). The impact of education on crime: international evidence. Leibniz Institute for Economic Research, 2, 49-55. 
Instituto de Pesquisa Econômica Aplicada. (2018). Atlas da Violência 2018. Brasília, DF: Autor.

Levitt, S. (1997). Using electoral cycles in police hiring to estimate the effect of police on crime. The American Economic Review, 87(3), 270-290.

Levitt, S. (2004). Understanding why crime fell in the 1990s: four factors that explain the decline and six that do not. Journal of Economic Perspectives, 18(1), 163-190.

Lima, J. A., Santos, J. R., Dal'Col, P. P. \& Silva, S. F. (2017). Teorias sociológicas sobre a criminalidade: análise comparativa de três teorias complementares. Revista Alamedas, 5(2), 77-102.

Loughran, T., Paternoster, R., Chalfin, A., \& Wilson, T. (2016). Can rational choice be considered a general theory of crime? Evidence from individuallevel panel data. Criminology, 54(1), 86-112.

Mallubhotla, A. (2013). An analysis of the relationship between employment and crime. The Park Place Economist, 21, 93-100.

Martins, J. S., \& Gomes, P. S. (2011). Identidade, desvio e reputação no gripo de pares. Antropológicas, 12, 67-73.

Mattos, M. (2018). Efeitos vizinhança e eficácia coletiva: a relevância do contexto na explicação sociológica. Revista Sociedade e Estado, 33(1), 295 301.

Messner, S. F. (1982). Poverty, inequality, and the urban homicide rate: some unexpected findings. Criminology, 20, 103-114.

Niknami, S. (2012). The effect of relative income on crime: evidence from micro-data (Doctoral Dissertation). Stockholm University, Stockholm, Sweden.

Ray, J., Baker, T. \& Caudy, M. (2020, February). Revisiting the generality of rational choice theory: Evidence for general patterns but differential effect across varying levels of psychopathy. Journal of Criminal Justice, 66, 101654, 1-11.

Scheeffer, F. (2013). Teoria da escolha racional: a evidenciação do homo economicus? Em Tese, 10(1), 28-43.

Secretaria da Segurança Pública e Administração Penitenciária. (2016). Manual de Interpretação Estatística. Goiânia, GO: SSPGO.

Secretaria-Geral da Presidência da República. (2018). Custos Econômicos da Criminalidade no Brasil. Brasília, DF: Relatório de Conjuntura.

Shaw, C. R. \& Mckay, H. D. (1942). Juvenile delinquency and urban areas. Chicago, IL: University of Chicago Press.

Shon, P. C. H., \& Barton-Bellessa, S. (2015). The Assumption of Rational Choice Theory in Alfred Adler's Theory of Crime: Unraveling and Reconciling the Contradiction in Adlerian Theory through Synthesis and Critique. Aggression \& Violent Behavior, 25(A), 95-103.

Silva, E. R. A. \& Oliveira, R. M. (2015). O adolescente em conflito com a lei e o debate sobre a redução da maioridade penal: esclarecimentos necessários. IPEA, 20, 2-41.

Stemen, D. (2007). Reconsidering incarceration: new directions for reducing crime. New York, NY: Vera Institute of Justice.

Strapazzon, C. L., \& Tramontina, R. (2015). As bases metodológicas/epistemológicas da Rational Choice Theory (RCT) e a análise econômica do Direito. Prisma Jurídico, 14(2), 107-138.

Sutherland, E. H. (1973). Development of the theory. In Schuessler. K. (Ed.), Edwin Sutherland on Analyzing Crime. Chicago, IL: University Press, 30-41.

Viapiana, L. T. (2006). Economia do crime: uma explicação para a formação do criminoso. Porto Alegre, RS: AGE Editora. 


\section{Sabrina Oliveira de Figueiredo}

https://orcid.org/0000-0002-3302-9489.

Mestra em Administração; Doutoranda do Programa de Pós-Graduação em Administração da Universidade Federal do Espírito Santo. E-mail: sab.figueiredo@gmail.com

\section{Larissa Alves Sincorá}

https://orcid.org/0000-0002-6590-7637

Mestra em Administração; Doutoranda do Programa de Pós-Graduação em Administração da Universidade Federal do Espírito Santo. E-mail: sincora.larissa@gmail.com

\section{Maria Clara de Oliveira Leite}

https://orcid.org/0000-0003-4980-6841

Mestra em Engenharia e Desenvolvimento Sustentável; Doutoranda do Programa de Pós-Graduação em Administração da Universidade Federal do Espírito Santo. E-mail: mariaclara.ol@hotmail.com

\section{Marcelo Moll Brandão}

https://orcid.org/0000-0002-8593-734X

Doutor em Administração; Professor permanente do Departamento de Administração e do Programa de Pós-Graduação em Administração da Universidade Federal do Espírito Santo. E-mail: mollmkt@gmail.com 